\title{
Needle Pulmonary Embolism in an Intravenous Drug User; A Case Report
}

\author{
Amr Arafat ${ }^{1}$, Mustafa Rady ${ }^{1}$, Mohamed Ebrahim ${ }^{1}$, and AHMED ELMAHROUK ${ }^{2}$ \\ ${ }^{1}$ Tanta University Faculty of Medicine \\ ${ }^{2}$ King Faisal Specialist Hospital and Research Centre - Jeddah
}

June 1, 2020

\begin{abstract}
Needle embolization in intravenous drug users to the lung is rarely reported in the literature. The management of these patients is controversial. We presented a male patient aged 33-year-old who presented with a broken needle in the left arm after trial of self-drug injection. During the trial to retrieve the needle, the needle embolized to the lung. Chest X-ray showed the needle in the right lower lobe, and the chest $\mathrm{CT}$ scan confirmed the diagnosis. The patient complained of non-specific right-side chest pain that was controlled with analgesics. The patient was managed conservatively and was discharged on antiplatelet therapy. After the 6-month follow-up, the patient was asymptomatic with no complication.
\end{abstract}

\section{Introduction:}

Central needle embolization in intravenous drug users is a rare event, with few cases reported in the literature (1). It is not known whether embolised needle has a predilection to a specific side or lobe. There is no consensus on how to manage these patients since the reported complications are very rare. The embolised needle may pass unnoticed because of the stigmata of abusing drugs in our community, yet, it may present later with pulmonary complications. Increasing the awareness of this rare entity could help the detection of more cases with more accurate monitoring of the long-term consequences. Foreign body pulmonary embolism was reporter in the literature (2-4); however, needle embolization is scarce.

We reported a case of needle embolization to the right lower lobe that occurred during the trial of the needle retrieval from the left cephalic vein.

\section{Case report:}

A 33-year-old male presented to our hospital with a broken needle in his left arm after a trial of self-drug injection. His first complaint was left shoulder pain where the needle was detected on shoulder X-ray after examination by orthopaedic surgeons.

Under fluoroscopic guidance, a trial of removal by the vascular surgeons was done (Figure 1); however, the needle was embolized. A chest X-ray showed the needle in the right lung. (Figure 2)

A chest contrast-enhanced CT scan was done and revealed the needle embedded in the right lower lung lobe. (Figure 4) There was no evidence of venous thromboembolism or infarction and no detectable pericardial effusion.

The decision was made to manage the case conservatively because the patient complained from chest pain localized to the right side, which was controlled by analgesics. The patient was discharged in low dose Aspirin, and there were no complications reported after six months of follow-up.

\section{Discussion:}


Intravenous drug users may experience needle breaking incidences during their self-injection. (5) The time taken for needles to migrate from soft tissues and embolize centrally can vary from a matter of a few hours to several days (6). Needle embolization to the lung is rarely reported in intravenous drug abusers, and most of the reported cases of needle embolization were iatrogenic embolization after the vascular intervention.

Richardson and colleagues performed a literature review of over 200 patients who had embolization of catheter fragments. They identified embolization to the lung periphery in eight cases; none of them required treatment. (7)

Needle breaking is common in intravenous drug users, but under-reported. In a survey among intravenous drug users, one-fifth of participants reported a needle breaking during drug injection, many of which could not be surgically or individually recovered. (5) Among eight patients of pulmonary needle embolism, Hart and co-workers reported seven cases with right lung embolization (8). In agreement with most of the reported embolization to the lung, our patient had embolization to the right lung.

Needle embolization to the heart was associated with pericarditis, endocarditis, and pulmonary abscess (9). However, most cases of needle embolization to the lung were not associated with complications. One patient with needle embolization to the lung presented with organized pneumonia with staph aureus (1).

Because of the stigmata of drug abuse, patients with broken needles may be reluctant to seek medical advice. Patients may present later with pulmonary complications such as infection or pneumothorax, and prior awareness of this condition could lead to the diagnosis of the cause. Migration of foreign bodies outside the lung has been previously reported (10), and needle embolization should be suspected in all pulmonary foreign bodies.

There is no standard medical management for pulmonary needle embolism reported in the literature. We discharged the patient on low dose Aspirin for fear of thrombosis on top of the foreign body. The role of antiplatelet medications in these cases is not known, and there are no recommendations against or with the use of them in needle pulmonary embolization. The patient remained symptom-free, and no complications were reported after six months of follow-up.

\section{Conclusion}

Pulmonary needle embolization is a rare entity. There is limited experience in the management of this condition. Our patient was managed safely without surgical retrieval of the needle, and antiplatelets may have a role in these cases.

\section{References:}

1. Eric J. Monroe, MD; Tina D. Tailor, MD; Michael F. McNeeley, MD; and Bruce E. Lehnert, MD Needle embolism in intravenous drug abuse Radiology Case Reports Volume 7, Issue 3, 2012

2. Rief P, Belaj K, Smaczny N, et al. Foreign body pulmonary embolism.Wien Klin Wochenschr . 2013;125(11-12):337-338. doi:10.1007/s00508-013-0375-6

3. Naito N, Abe M, Fukasawa M, et al Pulmonary embolism by a foreign body that migrated in the inferior vena cava during lumbar spine surgery Case Reports 2014;2014: bcr2014205423.

4. Bakir, D.A., Othman, Y.N. \& Taha, A.Y. Pulmonary bullet embolism following cardiac gunshot wound: case report of a bullet that traversed the heart twice. Cardiothorac Surg 28, 5 (2020). https://doi.org/10.1186/s43057-020-0017-5

5. Guy A. Norfolk Selena F. Gray. Intravenous drug users and broken needles-a hidden risk? Addiction. 2003 Aug;98(8):1163-6

6. Angelos, M. G., Sheets, C. A. \& Zych, P. R. (1986) Needle emboli to lung following intravenous drug abuse. Journal of Emergency Medicine, 4, 391-396. 
7. Richardson JD, Grover RL, Trinkle JK. Intravenous catheter emboli. Experiences with twenty cases and collective review. Am J Surg. 1974:128(6);722-727.

8. Hart BL, Newell JD 2nd, Davis M. Pulmonary needle embolism from intravenous drug abuse. Am J Emerg Med 1989;40(6):326-7.

9. Gyrtrup, H. J., Andreassen, K. H., Pedersen, J. H. \& Mortensen, L. B. (1989) Central embolization of needle fragment following intravenous drug abuse. British Journal of Addiction, 84, 103-105.

10. Arafat A; Taha A; Elkhouly A; Elgalad A. Can a foreign body migrate against natural body barriers? Annals of Pediatric Surgery. 13(3):157-159, JULY 2017

\section{Figures legends:}

Figure 1: Fluoroscopy of the left arm during the trial of needle retrieval showing the needle in cephalic vein around the shoulder joint

Figure 2: Chest X-ray, lateral view showing the needle in the lower lung zone

Figure 3: CT chest with 3D reconstruction showing the needle on the right side of the chest

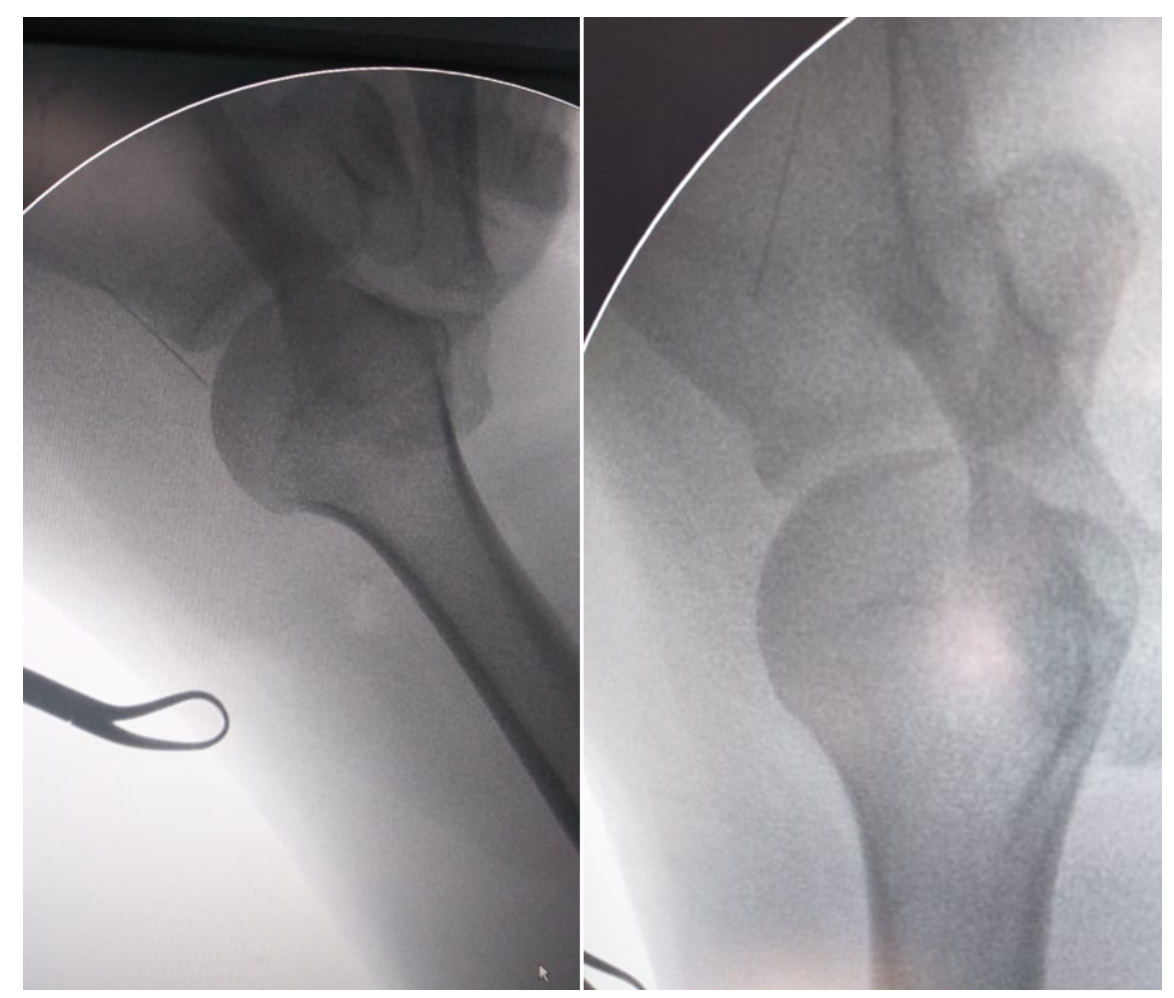



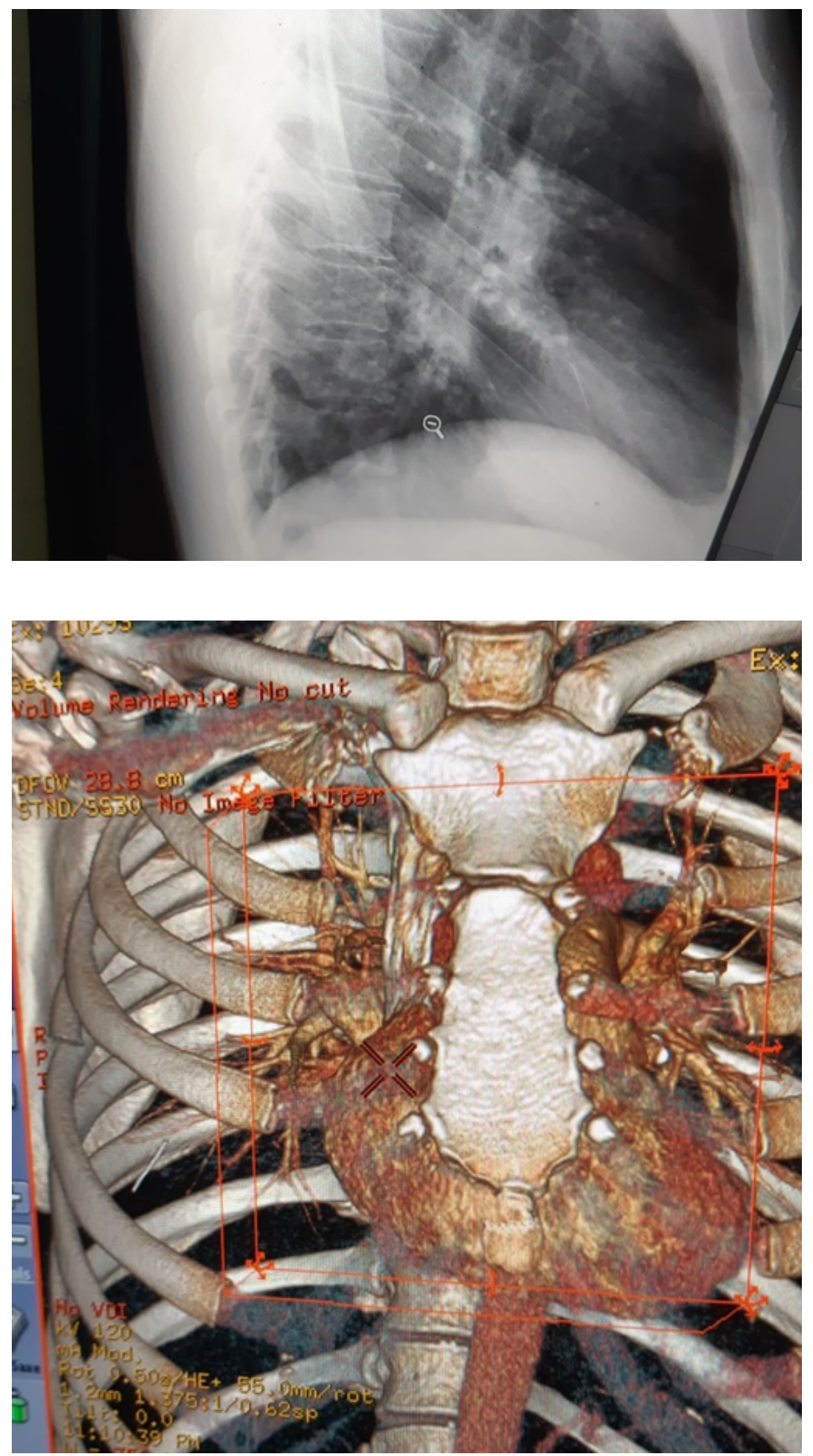